\title{
Sensibilidade ao Contraste de Freqüências Radiais em Crianças de 4 a 7 Anos e Adultos
}

\author{
Radial Frequency Contrast Sensitivity in Children of 4 to 7 Years Old and Adults
}

\author{
Natanael Antonio dos Santos ${ }^{*} a$, Valtenice França ${ }^{b} \&$ Maria Lucia de Bustamante Simas ${ }^{c}$ \\ ${ }^{a}$ Universidade Federal da Paraíba, João Pessoa, Brasil, ${ }^{b}$ Universidade de São Paulo, São Paulo, Brasil \\ ${ }^{c}$ Universidade Federal de Pernambuco, Recife, Brasil
}

\begin{abstract}
Resumo
O objetivo deste trabalho foi medir a sensibilidade ao contraste para frequiências radiais (FSCr) de 0,25 a $2 \mathrm{cpg}$ em crianças ( 4 a 7 anos) e adultos. Foram estimados limiares de contraste para 25 participantes (vinte crianças e cinco adultos jovens), utilizando o método psicofísico da escolha forçada. Os participantes apresentavam acuidade visual normal. Os resultados mostraram que as curvas (FSCr) de crianças de 4 a 7 anos melhoraram de forma significativa com a idade. Os resultados mostraram ainda que a FSCr de crianças de 7 anos é mais baixa do que a de adultos jovens. Estes resultados sugerem que o desenvolvimento da percepção visual de contraste para estímulos radiais ocorre gradualmente se prolongando além dos 7 anos.

Palavras-chave. Sensibilidade ao contraste; desenvolvimento visual; crianças; frequiência radial; método da escolha forçada.
\end{abstract}

\begin{abstract}
The aim of this work was to measure contrast sensitivity for radial frequencies (rCSF) in the range between 0.25-2 cpd in children ( 4 to 7 years of age) and young adults. Contrast thresholds were estimated for 25 participants (twenty children and five adults) using the psychophysical forced-choice method at low luminance levels. All the cts participants had normal acuity. The results showed that the curves (rCSF) of the 4, 5, 6 and 7 years old improved significantly with age. The results also showed that the rCSF for 7 year old children was lower than in young adults. These results suggest that the development of contrast sensitivity for radial stimuli increases gradually even after turning 7 years old.

Keywords: Contrast sensitivity; visual development; children; radial frequency; forced-choice method.
\end{abstract}

\section{Função de Sensibilidade ao Contraste e Percepção Visual}

A função de sensibilidade ao contraste (FSC) é definida na literatura como a recíproca da quantidade mínima de contraste necessária para detectar um objeto com uma dada freqüência espacial (Cornsweet, 1970). Além de ser um dos principais indicadores das funções visuais (Wilson, Levi, Maffei, Rovamo \& De Valois, 1990), ela descreve de forma eficiente o desempenho do sistema visual em níveis diferentes de contrastes, fornecendo, inclusive, o limite entre o mundo a baixo contraste, que é, entretanto, percebido, e o mundo a baixo contraste, que nunca se vê (Santos \& Simas, 2001). A FSC é uma medida clássica que permite descrever mecanismos sensoriais básicos, fornecendo uma das descrições mais completas do sistema visual (Adams \& Courage, 2002; Wilson et al., 1990). Neste contexto, a FSC se tornou um instrumento importante na análise clínica e teórica de estruturas da percepção visual

* Endereço para correspondência: Universidade Federal da Paraíba, Centro de Ciências Humanas Letras e Artes, Campus I, Departamento de Psicologia, Laboratório de Processamento Visual Humano, Cidade Universitária, João Pessoa, PB, 58051-900. Tel.: (83) 3216 7006; Fax: (83) 32167337. E-mail:natanael.santos@pesquisador.cnpq.br
(Adams \& Courage; Montés-Micó \& Ferrer-Blasco, 2001). Em termos gerais, a FSC determina o limiar de contraste em função da freqüência especial. Freqüência espacial é definida como o número de ciclos ou amplitudes máximas e mínimas de luminância por grau de ângulo visual (cpg). Já o contraste é definido como a diferença entre a modulação de luminância máxima e a mínima do estímulo dividido pela soma das duas.

\section{Sensibilidade ao Contraste e Desenvolvimento da Percepção Visual em Criança}

Estudos psicofísicos e eletrofisiológicos realizados ao longo das últimas décadas com recém-nascidos destacam a importância da FSC na investigação de mecanismos sensoriais básicos durante o desenvolvimento da percepção visual (Allen, Tyler \& Norcia, 1996; Atkinson, Braddick \& Braddick, 1974; Atkinson, Braddick \& Moar, 1977; Cannon Jr., 1983; Peterzell, Werner \& Kaplan, 1995). A maioria destes estudos relata que a FSC é muito pobre ao nascimento, melhorando rapidamente durante os primeiros meses (Allen et al.; A thinson et al; Bradley \& Freeman, 1982; J. P. Kelly, Borchert \& Teller, 1997; Peterzell et al.). Contudo, o processo de desenvolvimento da FSC se estende além dos primeiros meses podendo perdurar por vários anos (Adams \& Courage, 2002; Allen et al.; 
Arundale, 1978; Atkinson et al.; Benedek, Benedek, Kéri \& Janáky, 2003; Bradley \& Freeman, 1982; Cannon Jr.; Ellemberg, Lewis, Liu, \& Maurer, 1999; Peterzell et al.). Por exemplo, Bradley e Freeman mediram a FSC para crianças de 2 a 16 anos, utilizando uma variação do método psicofísico da escolha forçada entre duas alternativas e luminância média de $250 \mathrm{~cd} / \mathrm{m}^{2}$, e não encontraram diferenças significativas entre crianças de 8 anos e adultos. Por sua vez, Ellemberg et al. mediram a FSC para crianças de 4 a 7 anos, utilizando o método psicofísico dos limites e luminância média de $9 \mathrm{~cd} / \mathrm{m}^{2}$, e não encontraram diferenças significativas entre as crianças de 7 anos e adultos. Por outro lado, Adams e Courage mediram a FSC de crianças de 1 mês a 9 anos, utilizando estímulos do tipo grade senoidal impressos em car tões (Vistech Chart) e luminância média de $70 \mathrm{~cd} / \mathrm{m}^{2}$, e não encontraram diferenças significativas entre crianças de 9 anos e adultos. Finalmente, G. Benedek et al. mediram a FSC de crianças e adolescentes de 5 a 15 anos, utilizando o método psicofísico do ajuste e dois níveis de luminância $\left(0,09\right.$ e $\left.9,0 \mathrm{~cd} / \mathrm{m}^{2}\right)$, e não encontraram diferenças significativas entre crianças de 11-12 anos e adultos. Em linhas gerais, os estudos com grades senoidais mostram que a FSC melhora gradativamente com a idade e que o período de maturação do sistema visual da criança depende das condições de visualização e do método psicofísico empregado.

No presente estudo, mediu-se curvas de sensibilidade ao contraste para freqüências radiais (FSCr) para crianças de 4 a 7 anos e adultos jovens (19-22 anos), utilizando o método psicofísico da escolha forçada em nível baixo de luminância (luminância média de $0,7 \mathrm{~cd} / \mathrm{m}^{2}$ ). O objetivo principal foi caracterizar a existência de relações entre o desenvolvimento visual e as estimativas de sensibilidade ao contraste para freqüências radiais (FSCr). As pesquisas que determinaram a resposta do sistema visual humano para freqüências radiais são dispersas e raras com adultos (D. H. Kelly, 1982; D. H. Kelly \& Magnuski, 1975; Santos, Oliveira, Nogueira \& Simas, 2006; Simas \& Santos, 2002) e não encontramos nenhum estudo na literatura que relacionasse a sensibilidade ao contraste para freqüências radiais (ou estímulos simétricos com configurações semelhantes) ao desenvolvimento visual da criança. Mesmo, a primeira proposta de estudar padrões radiais em coordenadas polares ao invés de coordenadas cartesianas (grade senoidal) tendo sido surgida no início da década de 60, quando D. H. Kelly (1960) sugeriu este tipo de estímulo modulado pela função cilíndrica de Bessel.

\section{Método}

\section{Participantes}

Participaram do estudo 25 voluntários entre crianças (10 do sexo masculino e 10 do sexo feminino) e adultos (dois do sexo masculino e três do sexo feminino). Os mesmos foram divididos em cinco grupos com cinco participantes $[4$ anos $(M=4,3$ anos; $D P=3$ meses $), 5$ anos $(M=5,4$ anos; $D P=3$ meses), 6 anos $(M=6,4$ anos; $D P=2$ meses $), 7$ anos $(M=7,4$ anos; $D P=3$ meses $)$ e adultos jovens de $19-$ 22 anos $(M=21,3$ anos; $D P=14$ meses $M)]$. Os símbolos
$M$ e $D P$ representam a idade média e o desvio padrão de cada faixa etária, respectivamente. Todos apresentavam acuidade visual normal ou corrigida (20/20) e não tinham história de patologia ocular. A acuidade foi medida com a cartela de optotipos «E» de Rasquin. A participação na pesquisa ocorreu mediante assinatura de termo de consentimento livre e esclarecido conforme a Resolução $n^{\circ}$ 196/96 do Conselho Nacional de Saúde (Ministério da Saúde), que trata das diretrizes e normas de pesquisas envolvendo seres humanos. No caso das crianças, o termo foi assinado pelo responsável. Projeto aprovado pelo comitê de Ética Local (Centro de Ciências da Saúde).

\section{Equipamentos e Estímulos}

Os estímulos radiais foram gerados em um monitor de vídeo CLINTON MEDICAL monocromático de 21 polegadas, digital e de alta resolução controlado por um microcomputador. Uma cadeira foi fixada a $150 \mathrm{~cm}$ da tela do monitor de vídeo. A luminância média foi de $0,7 \mathrm{~cd} / \mathrm{m}^{2}$ ajustada por um fotômetro do tipo SPOT METTER, com precisão de um grau, ASAHI PENTAX. A luminância mínima foi de $0,5 \mathrm{~cd} / \mathrm{m}^{2}$ e a máxima de $0,9 \mathrm{~cd} / \mathrm{m}^{2}$. O ambiente do laboratório era cinza para melhor controle da luminância.

Foram utilizados estímulos circularmente simétricos com freqüências radiais de 0,25; 0,5; 1 e 2 ciclos por grau de ângulo visual, cpg (Figura 1). Estes eram circulares com um diâmetro de 7 graus de ângulo visual a $150 \mathrm{~cm}$ de distância da tela e foram gerados em tons de cinza e apresentados em tempo real no monitor. A primeira proposta de estudar padrões radiais em coordenadas polares ao invés de coordenadas cartesianas (grade senoidal) partiu de D. H. Kelly (1960), que sugeriu este tipo estímulo modulado pela função cilíndrica de Bessel. Outras informações sobre estes padrões podem ser encontradas na literatura (D. H. Kelly, 1982; D. H. Kelly \& Magnuski, 1975; Santos \& Simas, 2002; Santos, Simas \& Nogueira, 2004; Simas \& Santos, 2002).

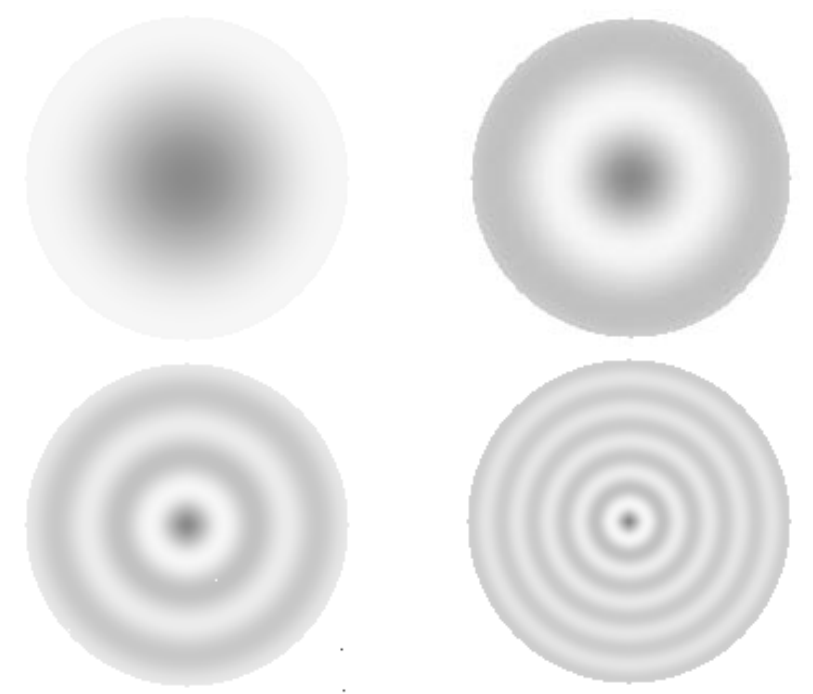

Figura 1. Exemplos de estímulos de freqüências radiais (acima, da esquerda para a direita, 0,25 e 0,5 cpg e embaixo, da esquerda para direita, 1 e 2 cpg). Estímulos originalmente calibrados para serem vistos a $150 \mathrm{~cm}$ de distância. 


\section{Procedimento}

As estimativas foram realizadas com o método psicofísico da escolha forçada (Santos et al., 2006; Wetherill \& Levitt, 1965). Este método se baseia no cálculo da probabilidade de acertos consecutivos por parte do participante, ou seja, em cerca de 100 apresentações de escolhas entre os dois estímulos (onde se exige três acertos consecutivos e um erro para mudar o contraste), a freqüência radial (estímulo de teste) foi percebida, por exemplo, em $79 \%$ das vezes pelo voluntário. O procedimento para medir o limiar para cada freqüência consistiu na apresentação sucessiva simples do par de estímulos e o participante teve que escolher dentre eles qual continha a freqüência radial. O outro estímulo (estímulo neutro) foi sempre um padrão homogêneo com luminância média de $0,7 \mathrm{~cd} / \mathrm{m}^{2}$. O critério adotado para variar o contraste de cada freqüência radial testada foi o de três acertos consecutivos para decrescer uma unidade e um erro para acrescer da mesma unidade (0,08\%).

Durante cada sessão experimental foi apresentada uma seqüência de estímulos iniciada com um sinal sonoro acompanhado imediatamente pela apresentação do primeiro estímulo por $2 \mathrm{~s}$, seguido de um intervalo entre estímulos de $1 \mathrm{~s}$ e pela apresentação do segundo estímulo por $2 \mathrm{~s}$ e da resposta do participante. A ordem de apresentação dos estímulos era aleatória. Se a resposta do participante fosse correta, era seguida por outro sinal sonoro e um intervalo de $3 \mathrm{~s}$ para a seqüência se repetir. O sinal sonoro que indicava o início da apresentação do par de estímulos e o que indicava a escolha correta eram diferentes. A duração da sessão experimental variou entre 5 e 10 minutos, dependendo dos erros e acertos do participante até proporcionarem um total de cinco reversões conforme requerido para o final automático da mesma.

Cada um dos pontos (ou frequiências) da curva de limiar de contraste foi estimado pelo menos duas vezes (duas sessões experimentais), em dias diferentes, por cada um dos participantes. Em média, dez curvas foram mensuradas para cada grupo de voluntários totalizando 40 sessões experimentais. Todas as medições ocorreram à distância de $150 \mathrm{~cm}$, com visão binocular.

Os participantes foram instruídos a pressionar o botão do lado esquerdo do mouse quando a freqüência radial era apresentada primeiro e o botão do lado direito quando era apresentada em segundo lugar, isto é, após o estímulo neutro. Os experimentos só começaram quando o experimentador certificou-se que todos os participantes entenderam e responderam conforme as instruções.

\section{Resultados}

A Figura 2 mostra a FSCr (função de sensibilidade ao contraste para freqüências radiais) média para cada uma das cinco faixas etárias (crianças de 4 a 7 anos e adultos jovens). Os limiares de contrastes são apresentados em função da freqüência radial. A sensibilidade ao contraste (FSCr) é o inverso do limiar de contraste (1/FSCr). Isto é, quanto menor o limiar de contraste maior a sensibilidade do sistema visual humano e vice-versa. Assim, os me- nores valores de limiares correspondem aos maiores valores de sensibilidade ao contraste.

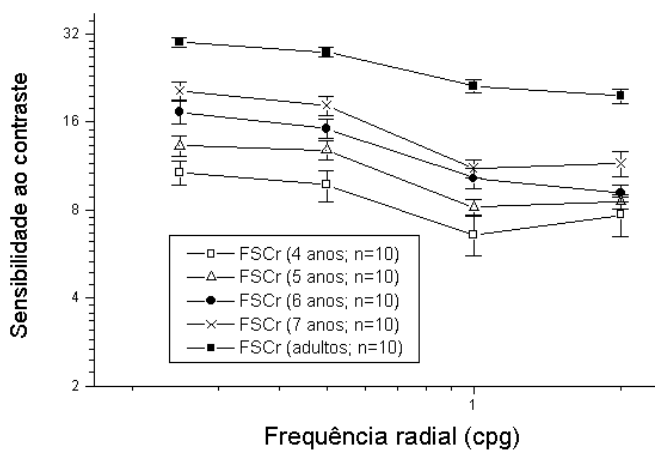

Figura 2. Funções de sensibilidade ao contraste de crianças de 4, 5, 6 e 7 anos e adultos para freqüências radiais (FSCr). $\mathrm{O} n$ representa o número de curvas mensuradas para cada faixa etária. As linhas verticais mostram o erro padrão da média para cada frequiência $(0,25 ; 0,5 ; 1$ e $2 \mathrm{cpg})$.

A sensibilidade máxima ocorreu na freqüência radial de 0,25 cpg para todas as idades (Figura 2). Entretanto, a sensibilidade das crianças de 4 anos na freqüência radial de 0,25 cpg foi da ordem de 1,2 vezes menor do que a das crianças de 5 anos. Já a sensibilidade das crianças de 5 anos foi da ordem de 1,3 vezes menor do que a das crianças de 6 anos que, por sua vez, foi da ordem de 1,2 menor do que a das crianças de 7 anos. Já para as crianças de 7 anos, a sensibilidade ao contraste na freqüência radial de 0,25 cpg foi da ordem de 1,5 menor do que a dos adultos. As diferenças encontradas entre as idades para as demais freqüências $(0,5 ; 1,0$ e $2,0 \mathrm{cpg})$ foram semelhantes ao que ocorreu na freqüência de $0,25 \mathrm{cpg}$.

A comparação da idade versus freqüências radiais através da ANOVA permitiu observar efeito principal significativo de idade, $\mathrm{F}_{(4,1410)}=188,43(p<0,001)$, um efeito principal significativo de freqüência radial, $F_{(3,1410)}=123,24$ $(p<0,001)$, e uma interação significativa de idade versus freqüência radial, $\mathrm{F}_{(12,1410)}=36,14(p<0,001)$. O teste HSD de Tukey foi usado para comparações post-hoc. As análises mostraram diferenças estatísticas entre a sensibilidade ao contraste (FSCr) de crianças de 4 e 5 anos $(p<0,001)$, 5 e 6 anos $(p<0,001), 6$ e 7 anos $(p<0,001)$ e entre 7 anos e adultos jovens $(p<0,001)$. Estes resultados mostram que a FSCr melhora de forma significativa com o aumento da idade e que a FSCr de crianças até 7 anos é diferente da FSCr de adultos. Isto é, a maturação da FSCr se estende para idades maiores do que aquelas utilizadas nesta pesquisa.

\section{Discussão}

Os resultados do presente estudo mostraram que o método psicofísico da escolha forçada, tradicionalmente utilizado nas pesquisas com adultos e grades senoidais, pode ser utilizado de forma confiável para medir a sensibilidade ao contraste de freqüências radiais (FSCr) de crianças a partir dos 4 anos. A Figura 2 demonstra que a FSCr das crianças de 4 anos apresenta um perfil geral muito seme- 
lhante às curvas de sensibilidade de crianças de 5, 6 e 7 anos e adultos jovens (19-22 anos). A diferença entre a FSCr das crianças de 4 anos e as outras idades pode ser relacionada ao domínio da sensibilidade ao contraste que aumenta gradativamente com o aumento da idade. Este dado é importante porque a medição da FSC com método psicofísico tem uso limitado quando lida com crianças em idades pré-escolares de 3 a 5 anos (Richman \& Lyons, 1994). A limitação é tal que Richman e Lyons só conseguiram medir a FSC de crianças nesta faixa etária utilizando um procedimento simples, no qual os estímulos eram apresentados em cartões (Vistech Chart) e a criança precisava apenas olhar ou apontar na direção do cartão que continha a grade senoidal. Em outras palavras, os métodos psicofísicos geralmente utilizados com adultos não são efetivos quando testam crianças pré-escolares. Outras informações acerca do uso da FSC em crianças pré-escolares podem ser encontradas em Richman e Lyons.

As curvas de sensibilidade ao contraste para freqüências radiais (FSCr) mostram que a faixa de maior sensibilidade ocorreu na freqüência radial de $0,25 \mathrm{cpg}$ para todas as idades (Figura 2). Isto significa que embora a FSCr melhore com o aumento da idade, a faixa de freqüência radial em que o sistema visual é mais sensível continua em 0,25 cpg. Entretanto, os resultados demonstram diferenças significativas no processo de maturação da FSCr entre todas as idades $(p<0,001)$. Isto é, a FSCr melhora gradativamente com o aumento da idade. Aumento na sensibilidade ao contraste relacionado ao desenvolvimento já era esperado, pois vários estudos relatam dados nesta direção com métodos psicofísicos diferentes, só que utilizando grade senoidal vertical (Adams \& Courage, 2002; Arundale, 1978; Bradley \& Freeman, 1982; Benedek et al., 2003; Ellemberg et al., 1999).

Os resultados mostram ainda que o desempenho (FSCr) das crianças de 4, 5, 6 e 7 anos é estatisticamente diferente do desempenho de adultos $(p<0,001)$. Por exemplo, as crianças de 7 anos foram as que mais se aproximaram do desempenho dos adultos, ainda assim, elas precisaram em média da ordem 1,$5 ; 1,5 ; 2$, o e 1,7 vezes mais contraste do que os adultos para detectar as freqüências radiais de 0,25; 0,5 ; 1 e $2 \mathrm{cpg}$, respectivamente. As diferenças foram maiores nas freqüências radiais de 1 e $2 \mathrm{cpg}$ (Figura 2).

Estes achados com freqüências radiais são diferentes daqueles discutidos no início por Bradley e Freeman (1982) e Ellemberg et al. (1999) para grades senoidais verticais. Pois, estes autores relataram que a FSC de crianças alcança a maturação por volta dos 7-8 anos, enquanto os nossos resultados mostram que a FSCr de crianças de 7 anos ainda não atingiu o processo de maturação do adulto. Entretanto, esta é uma comparação indireta, pois estes autores utilizaram condições diferentes, por exemplo, estímulos espaciais (grades senoidais) ao invés de estímulos de freqüências radiais. Grade senoidal é um estímulo de freqüência espacial clássico cuja luminância mínima e máxima varia em torno da luminância média em uma direção no espaço de acordo com o seno e/ou cosseno, sendo facilmente definida em um sistema de coordenadas cartesianas. Ao pas- so que o estímulo de freqüência radial é um padrão cuja luminância mínima e máxima varia em torno da luminância média na direção radial (ou seja, a luminância varia de acordo com o seno ou o cosseno do centro para a periferia nesse tipo de padrão), o que o torna um estímulo facilmente definido em um sistema de coordenadas polares com a origem do sistema de coordenadas no centro do estímulo (D. H. Kelly, 1982; D. H. Kelly \& Magnuski, 1975; Simas \& Santos 2002). As vantagens de estímulos radiais são que os mesmos apresentam um centro de fixação central claro (Figura 1) e segundo alguns autores estes estímulos são naturalmente mais apropriados para estudar o sistema visual considerando a simetria aproximadamente circular da retina (D. H. Kelly, 1960; D. H. Kelly \& Magnuski, 1975). Além disto, estudos psicofísicos (Santos \& Simas, 2002; Santos et al., 2004; Simas \& Santos; Wilson, Wilkinson \& Asaad, 1997; Wilson \& Wilkinson, 1998) e neurofisiológicos (Gallant, Connor, Rakshit, Lewis \& van Essen, 1996; Heywood, Gadotti \& Cowey, 1992; Merigan, 1996; Wilkinson et al., 2000) sugerem que estímulos visuais do tipo grade senoidal em coordenadas cartesianas são processados na área visual V1 (córtex visual primário) e que padrões radiais em coordenadas polares são processados por áreas visuais extra-estriados V4 e córtex ínfero-temporal (IT).

Em linhas gerais, os resultados mostraram que a FSCr varia em função do desenvolvimento e que estudos com padrões espaciais com configurações diferentes podem determinar quais estímulos são mais adequados para caracterizar a resposta do sistema visual. Podem ainda demonstrar de que maneira o processo de desenvolvimento e a maturação da FSC se relacionam com as vias ou áreas visuais sensoriais. Isto considerando a hipótese que estímulos em coordenadas cartesianas e polares são processados por áreas visuais distintas (Gallant et al., 1996; Heywood et al., 1992; Merigan, 1996; Wilkinson et al., 2000; Wilson \& Wilkinson, 1998). É nesta perspectiva que prosseguiremos com as nossas investigações.

\section{Referências}

Adams, R. J., \& Courage, M. L. (2002). Using a single test to measure human contrast sensitivity from early childhood to maturity. Vision Research, 42, 1205-1210.

Allen, D., Tyler, C. W., \& Norcia, A. M. (1996). Development of grating acuity and contrast sensitivity in the central and peripheral visual field of the human infant. Vision Research, 36(13), 1945-1953.

Arundale, K. (1978). An investigation into the variation of human contrast sensitivity with age and ocular pathology. British Journal of Ophthalmology, 62, 213-215.

Atkinson, J., Braddick, O., \& Braddick, F. (1974). Acuity and contrast sensitivity of infant vision. Nature, 247, 403-404.

Atkinson, J., Braddick, O., \& Moar, K. (1977). Contrast sensitivity of the human infant for moving and static patterns. Vision Research, 17, 1045-1047.

Benedek, G., Benedek, K., Kéri, S., \& Janáky, M. (2003). The scotopic low-frequency spatial contrast sensitivity develops in children between the ages of 5 and 14 years. Neuroscience Letters, 345, 161-164. 
Bradley, A., \& Freeman, R. D. (1982). Contrast sensitivity in children. Vision Research, 22, 953-959.

Cannon, M. W., Jr. (1983). Contrast sensitivity: Psychophysical and evoked potential methods compared. Vision Research, 23, $87-95$.

Cornsweet, T. N. (1970). Visual perception. New York: Academic Press.

Ellemberg, D., Lewis, T. L., Liu, C. H., \& Maurer, D. (1999). Development of spatial and temporal vision during childhood. Vision Research, 39, 2325-2333.

Gallant, J. L., Connor, C. E., Rakshit, S., Lewis, J. W., \& van Essen, D. C. (1996). Neural responses to polar, hyperbolic, and cartesian gratings in area $\mathrm{V} 4$ of the macaque monkey. Journal of Neurophysiology, 76, 2718-2739.

Heywood, C. A., Gadotti, A., \& Cowey, A. (1992). Cortical area $\mathrm{V} 4$ and its role in the perception of color. Journal of Neuroscience, 12, 4056-4065.

Kelly, D. H. (1960). J stimulus patterns for vision research. Journal of the Optical Society of America, 50, 1115-1116.

Kelly, D. H. (1982). Motion and vision: IV. Isotropic and anisotropic spatial response. Journal of the Optical Society of America, 72, 432-439.

Kelly, D. H., \& Magnuski, H. S. (1975). Pattern detection and the two dimensional Fourier transform: Circular targets. Vision Research, 15, 911-915.

Kelly, J. P., Borchert, K., \& Teller, D. Y. (1997). The development of chromatic and achromatic contrast sensitivity in infancy as tested with the sweep VEP. Vision Research, 37(15), 20572072 .

Merigan, W. H. (1996). Basic visual capabilities and shape discrimination after lesions of extrastriate area $\mathrm{V}_{4}$ in macaques. Visual Neuroscience, 13, 51-60.

Montés-Micó, R., \& Ferrer-Blasco, T. (2001). Contrast sensitivity function in children: Normalized notation for the assessment and diagnosis of diseases. Documenta Ophthalmologica, 103, 175-186.

Peterzell, D. H., Werner, J., \& Kaplan, P. S. (1995). Individual differences in contrast sensitivity functions: Longitudinal study of 4-, 6- and 8-month-old human infants. Vision Research, 35(7), 961-979.

Richman, J. E., \& Lyons, S. (1994). A forced choice procedure for evaluation of contrast sensitivity function in preschool children. Journal of the American Optometric Association, 65(12), 859-864.

Santos, N. A., \& Simas, M. L. B. (2001). Percepção e processamento visual da forma: Discutindo modelos teóricos atuais. Psicologia: Reflexão \& Crítica, 14(1), 157-166.

Santos, N. A., \& Simas, M. L. B. (2002). Percepção e processamento visual da forma em humanos: Filtros de frequiências radiais de 1 e $4 \mathrm{cpg}$. Psicologia: Reflexão E Crítica, 15(2), 383-391.

Santos, N. A., Simas, M. L. B., \& Nogueira, R. M. T. B. L. (2004). Processamento visual da forma em humanos: Curvas de limiar de contraste para padrões circularmente simétricos. Psicologia: Reflexão e Crítica, 17(2), 287-294.

Santos, N. A., Oliveira, A. B., Nogueira, R. M. T. B. L., \& Simas, M. L. B. (2006). Mesopic radial frequency contrast sensitivity function for young and older adults. Brazilian Journal of Medical and Biological Research, 39(6), 791-794.

Simas, M. L. B., \& Santos, N. A. (2002). Contrast sensitivity to radial frequencies modulated by $\mathrm{Jn}$ and jn bessel profiles. Brazilian Journal of Medical and Biological Research, 3, 13571366
Wetherill, G. B., \& Levitt, H. (1965). Sequential estimation of points on a psychometric function. The British Journal of Mathematical and Statistical Psychology, 48, 1-10.

Wilkinson, F., James, T. W., Wilson, H. R., Gati, J. S., Menon, E. S., \& Goodale, M. A. (2000). An fMRI study of the selective activation of human extrastriate form vision areas by radial and concentric gratings. Current Biology, 10, 1455-1458.

Wilson, H. R., Levi, D., Maffei, L., Rovamo, J., \& De Valois, R. (1990). The perception of form: Retina to striate cortex. In S. W. Spillmann \& J. S. Werner (Eds.), Visual perception: The Neurophysiological Foundation (pp. 231-271). New York: Academic Press.

Wilson, R. W., Wilkinson, F., \& Asaad, W. (1997). Concentric orientation summation in human form vision. Vision Research, 37, 2325-2330.

Wilson, H. R., \& Wilkinson, F. (1998). Detection of global structure in glass patterns: Implications for form vision. Vision Research, 38, 2933-2947.
Recebido: $31 / 10 / 2006$ $1^{a}$ revisão: 04/05/2007 $2^{a}$ revisão: $27 / 05 / 2007$ Aceite final: $31 / 05 / 2007$ 